\title{
PROCEDURE FOR DETERMINING THE RELIABILITY OF POWER SUPPLY TO CONSUMERS IN ELECTRICAL LAYOUTS CONTAINING MULTI-CONTACT SWITCHING SYSTEMS
}

Key words: reliability of power supply to consumers; reliability calculation procedure; multi-contact switching systems.

\section{Authors' personal details}

Alexander Vinogradov, ORCID: 0000-0002-8845-9718, Candidate of Technical Sciences, Associate Professor, head of the Laboratory of Electric Power Supply and Heating Systems, Federal Scientific Agroengineering Center VIM, 109428, Moscow, 1st Institutsky proezd, 5, bldg 1, e-mail: winaleksandr@rambler.ru.

Multi-contact switching systems are switching devices that have two or more contact groups that are controlled independently. The multi-contact switching systems used in $0,4 \mathrm{kV}$ electric grids enable power supply layouts to be changed flexibly by sectioning and reserving various parts of power lines. Points where multi-contact switching systems are installed in the electrical grid are determined by the reliability requirements of the power supply to consumers and based on the adequate sensitivity of the power grid protection against emergency modes. The procedure of determining the reliability of electric power supply to consumers in the electrical layouts containing multi-contact switching systems is described below. First, the potential points for installing multi-contact switching systems in the grid are found. The power grid is divided into sections. The reliability indices are identified for each section of the power grid. The reliability of power supply to consumers is calculated taking into account different scenarios in the electrical grid and with an option to reserve the power to consumers. Reliability

(C) Виноградов А.В. indices are calculated for each group of consumers connected to the corresponding sections of the power grid. To this end, consumers are divided into groups connected to the corresponding sections of the power grid, and their characteristics are determined. The grid layout equivalents are designed to calculate no failure operation probability in the electrical layout for each consumer group, then the probability and time of no-failure operation is calculated for each layout. Interruptions in power supply and under-supply costs for each group of consumers are determined; the total damage is calculated for the grid equivalents. The grid equivalents are compared in terms of reliability indices. The calculation procedure described in the paper showed that the reliability of power supply to consumers with multi-contact switching systems in the electrical layout is more than 7 times higher than the reliability of the radial power supply layout. The procedure can be used in the design of intelligent electrical grids based on the multi-contact switching systems.

УДК 629.11

DOI: 10.31563/1684-7628-2020-54-2-84-89

Е.А. Максимов, Е.П. Устиновский

\section{ИССЛЕДОВАНИЕ ВЛИЯНИЯ ТИПА ВЫЛЕТА КОЛЕСА СЕЛЬСКОХОЗЯЙСТВЕННОЙ ТЕХНИКИ НА ИЗГИБ ПОСАДОЧНОЙ ПОЛКИ ОБОДА}

Ключевые слова: типы вылета трактора и комбайна; нормальный; отрицательный; положительный; посадочная полка обода колеса.

Введение. В зависимости от способа производства дисков колес тракторов и комбайнов все диски имеют строго типизированную форму и размеры. При подборе дисков колес тракторов следует учитывать такой параметр, как вылет.

При эксплуатации колес тракторов и комбайнов на него могут быть установлены колеса, имеющие нулевой, положительный и отрицательный вылеты.

Вылет выбирается в зависимости от конструкции автомобиля. Положительный и нулевой вылеты обычно используются в легковых автомобилях, а отрицательный вылет - в грузовых. 
Под вылетом (ЕТ) понимается расстояние между плоскостью, проходящей через середину посадочной полки обода, и привалочной плоскостью [1-9].

Рассмотрим влияние типа вылета на изгиб посадочной полки обода.

Известно, что на изгиб посадочной полки обода влияют факторы: давление воздуха в шине, вертикальная сила, а также тип вылета.

При этом данные факторы воздействуют на изгиб посадочной полки обода различно [10 $13]$.

Влияние нагрузки, обусловленной давлением воздуха в шине на изгиб посадочной полки обода, хорошо изучено $[14,15]$. Но влияние вертикальной нагрузки и типа вылета на изгиб посадочной полки обода до настоящего времени изучено недостаточно полно. Однако влияние данных факторов на изгиб посадочной полки обода необходимо знать для более точного расчета напряженно-деформированного состояния в диске колеса [16-18].

Цели исследования - провести анализ факторов, влияющих на изгиб посадочной полки обода; разработать методику расчета влияния вертикальной нагрузки и типа вылета на изгиб посадочной полки обода.

Методы исследований. Рассмотрим изгиб посадочной полки обода под действием вертикальной силы диска колеса с положительным вылетом (рисунок 2). На рисунках 2, 3, 4 изображение диска и обода повернуто по отношению к аналогичному изображению, представленному на рисунке 1.

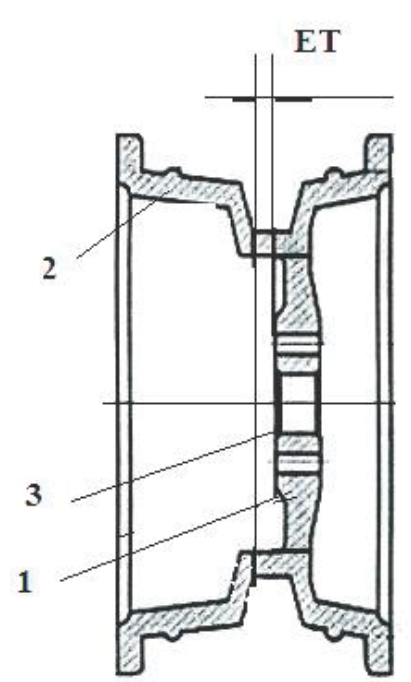

a)

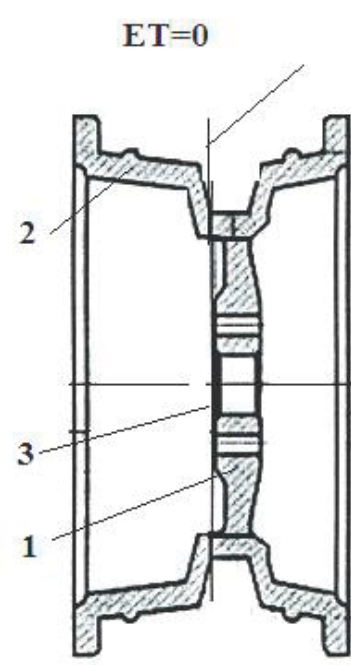

б)

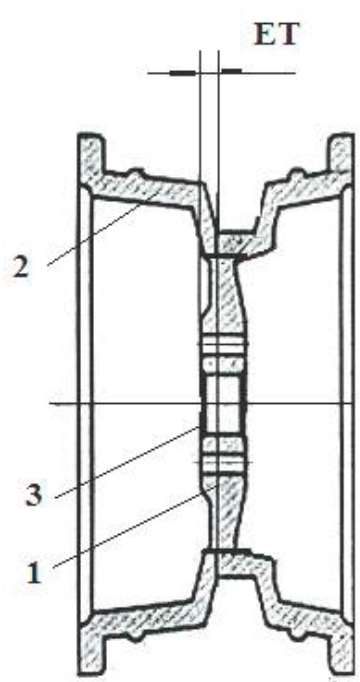

B)

Рисунок 1

Схема расположения вылета в диске колеса:

1 - диск, 2 - обод, 3 - привалочная плоскость, положительный вылет (а), нулевой вылет (б), отрицательный вылет (в)

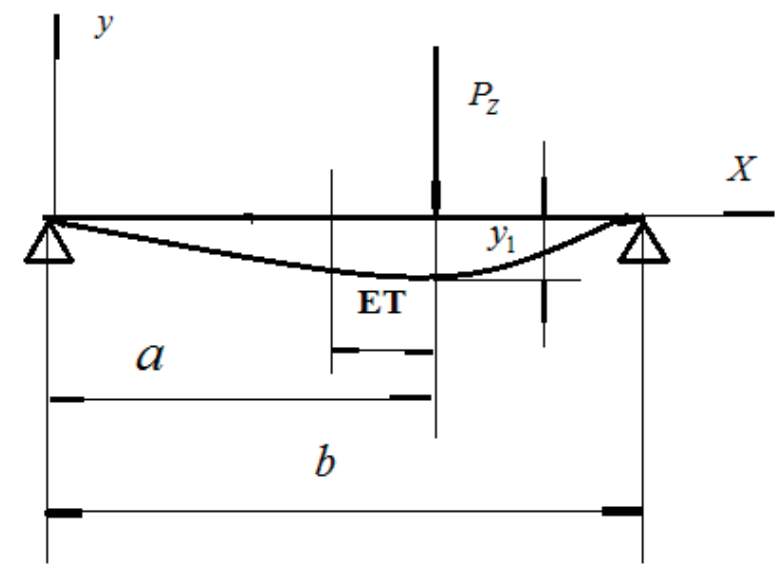

Рисунок 2

Схема диска колеса с положительным вылетом (ЕT)

Изгибающие моменты под действием вертикальной силы для правого и левого участков ширины посадочной полки обода определим по формулам

$$
\begin{gathered}
M_{1}=P_{Z} \frac{a}{b} X, \\
M_{2}=P_{Z} \frac{a}{b} X-P_{Z}(X-a),
\end{gathered}
$$


где $P_{Z}-$ вертикальная сила, $a$ - расстояние от закраины обода до места приложения вертикальной силы, $b$ - ширина посадочной полки обода.

После интегрирования получим

$$
\begin{gathered}
y_{1}=\frac{P_{Z}}{E I_{X}}\left(\frac{a}{b} \frac{X^{3}}{6}+C_{1} X+C_{2}\right), \\
y_{2}=\frac{P_{Z}}{E I_{X}}\left(\frac{a}{b} \frac{X^{3}}{6}-\frac{X^{3}}{6}+a \frac{X^{2}}{2}+C_{3} X+C_{4}\right),
\end{gathered}
$$

где $E I_{X}-$ жесткость диска.

Определим постоянные интегрирования в уравнениях (3.63) и (3.64) из следующих граничных условий:

$$
\begin{gathered}
\text { при } X=0, y_{1}=0, \\
\text { при } X=a, y_{1}=y_{2}, \\
\text { при } X=a, y_{1}{ }^{*}=y_{2}{ }^{*}, \\
\text { при } X=b, y_{2}=0 .
\end{gathered}
$$

Определим постоянные интегрирования из граничных условий

$$
\begin{gathered}
C_{1}=\frac{a}{6 b}\left(3 a b-2 b^{2}-a^{2}\right), \\
C_{2}=0, \\
C_{3}=-\frac{a}{6 b}\left(2 b^{2}+a^{2}\right), \\
C_{4}=\frac{a^{3}}{6} .
\end{gathered}
$$

Подставляя (9)-(12) в (3), (4), получим

$$
\begin{gathered}
y_{1}=\frac{P_{Z}}{E I_{X}}\left[\frac{b-a}{b} \frac{X^{3}}{6}+\right. \\
\left.+\frac{a X}{6 b}\left(3 a b-2 b-a^{2}\right)\right] \\
y_{2}=\frac{P_{Z}}{E I_{X}}\left[\frac{b-a}{b} \frac{X^{3}}{6}-\frac{a X^{2}}{2}-\right. \\
\left.-X \frac{a}{6 b}\left(2 b^{2}+a^{2}\right)+\frac{a^{3}}{6}\right]
\end{gathered}
$$

В зависимостях (13) и (14) максимальная величина изгиба обода будет наблюдаться в точке приложения силы $(X=a)$ :

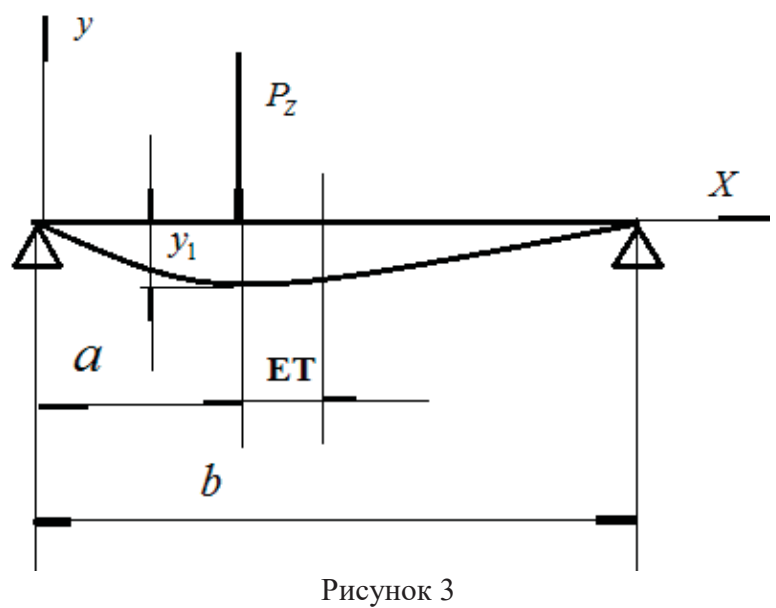

Схема диска колеса при отрицательном вылете (ЕT)

$$
\begin{aligned}
& y_{1}=\frac{P_{Z} a^{2}}{E I_{X} b}(b-a)^{2}, \\
& y_{2}=\frac{P_{Z} a^{2}}{E I_{X} b}(b-a)^{2} .
\end{aligned}
$$

При приложении вертикальной силы по середине ширины обода (нулевой вылет) уравнения (15), (16) примут вид

$$
\begin{aligned}
& y_{1}^{*}=\frac{P_{Z} b^{3}}{48 E I_{X}}, \\
& y_{2}^{*}=\frac{P_{Z} b^{3}}{48 E I_{X}} .
\end{aligned}
$$

Для диска колеса с отрицательным и нулевым типами вылета зависимости (17-18) будут иметь аналогичный характер.

Рассмотрим изгиб посадочной полки обода под действием вертикальной силы для отрицательного и нулевого вылета (рисунки 3,4 ).

Диск колеса трактора представлен на рисунке 5.

Обсуждение результатов. Расчет величины изгиба обода под действием вертикальной силы для колес тракторов и комбайнов представлен в таблице 1.

Анализ данных, представленных в таблице 1, показал, что максимальная величина изгиба обода наблюдается для нормального типа вылета. Для положительного и отрицательного типов вылета величина изгиба обода меньше, чем для нормального. Относительная величина расхождения между $y_{1}$ и $y_{1}{ }^{*}$ : для колеса составляет $\Delta=70 \%$, для колеса DW10-660 $\Delta=65 \%$, для колеса $385 / 65 \mathrm{R} 22.5 \Delta=60 \%$, для колеса $4,5 \mathrm{E}-16$ $\Delta=45 \%$, для колеса W8-16 $\Delta=30 \%$.

В целом можно отметить, что применение положительного и отрицательного вылетов при сборке диска колеса снижает величину максимального изгиба обода по сравнению с изгибом обода при нормальном типе вылета.

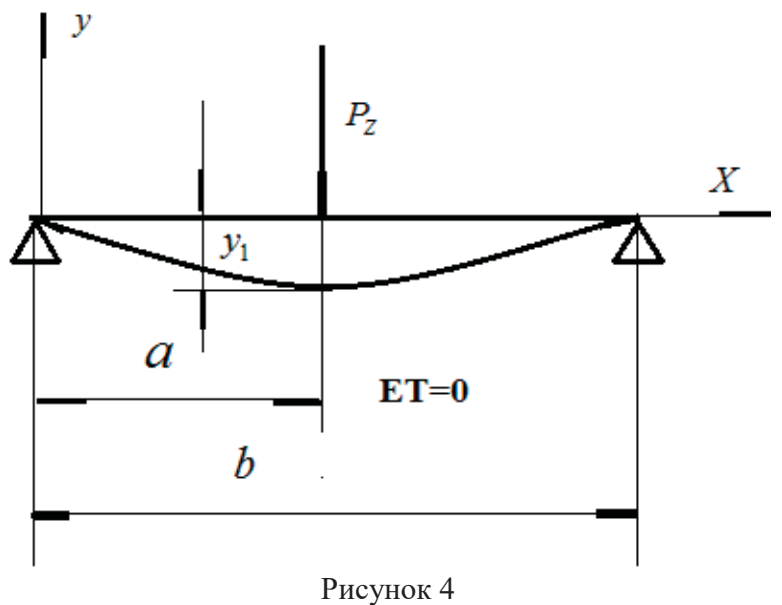

Схема диска колеса при нулевом вылете $(\mathrm{ET}=0)$ 


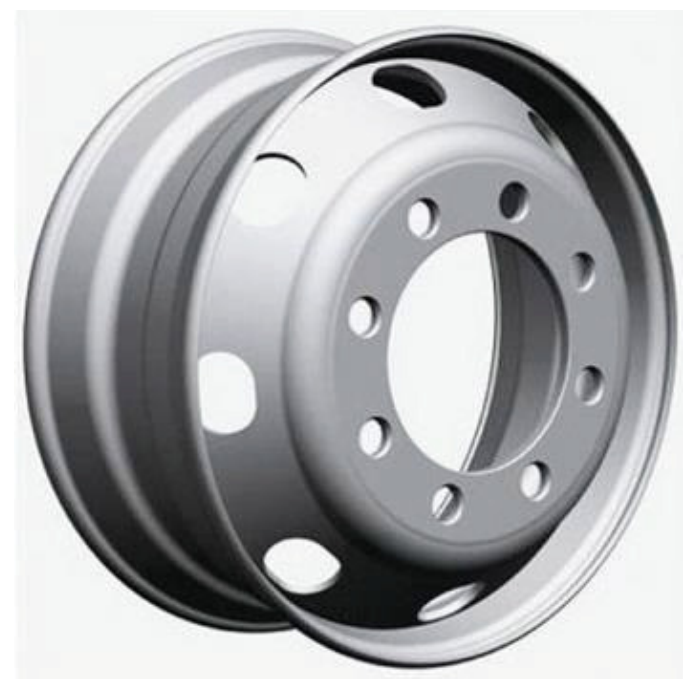

Рисунок 5

Диск колеса трактора

Таблица 1 Расчет величины изгиба обода под действием вертикальной силы для колес тракторов и комбайнов

\begin{tabular}{|c|c|c|c|c|c|c|}
\hline \multicolumn{4}{|c|}{ Тип вылета } & Нормальный & \multicolumn{2}{|c|}{ Положительный и отрицательный } \\
\hline \multirow{2}{*}{ Марка колеса } & $P_{Z}$ & $b$ & величина вылета & $y_{1}{ }^{*}=y_{2}{ }^{*}$ & $y_{1}$ & $\Delta$ \\
\hline & $\mathrm{H}$ & дюйм & MM & MM & MM & $\%$ \\
\hline DB14L-38* & 46000 & 10 & 120 & 0,5 & 0,15 & 70 \\
\hline DW10-660** & 30000 & 11 & 165 & 0,6 & 0,21 & 65 \\
\hline $385 / 65 \mathrm{R} 22.5$ & 20000 & 11,75 & 120 & 0,4 & 0,16 & 60 \\
\hline $4,5 \mathrm{E}-16 * * *$ & 7500 & 10 & 25 & 0,2 & 0,11 & 45 \\
\hline W8-16 & 1700 & 11 & 4 & 0,55 & 0,25 & 30 \\
\hline
\end{tabular}

В таблице 1: DB14L-38* для тракторов МТ3-80, DW10-660** для тракторов K-701, 4,5E-16*** колеса для тракторов Т30, Т$40 \mathrm{M}$, комбайнов Нива, Колос, $\Delta$ - относительная величина расхождения между $y_{1}$ и $y_{1}{ }^{*}$.

Выводы: 1. Проведен анализ различных факторов: давления воздуха в шине, вертикальной нагрузки и типа вылета на изгиб посадочной полки обода.

2. Разработана методика расчета влияния вертикальной нагрузки и типа вылета на изгиб посадочной полки обода.

3. Показано, что максимальная величина изгиба обода наблюдается для нормального типа вылета. Для положительного и отрицательного типов вылета величина изгиба обода меньше, чем для нормального.

4. Установлено, что применение положительного и отрицательного вылетов при сборке диска колеса снижает величину максимального изгиба обода по сравнению с изгибом обода при нормальном типе вылета.

\section{Библиографический список}

1. Вахламов, В.К. Автомобили, конструкции и элементы расчета [Текст] / В.К. Вахламов. М.: Академия, 2006. 408 с.

2. Стуканов, В.А. Устройство автомобилей [Текст] / В.А. Стуканов. М.: Форум, 2017. 496 с.

3. Чудаков, Е.А. Теория автомобиля [Текст] / Е.А. Чудаков. М.: Машиностроение, 1990. $344 \mathrm{c}$.

4. Гаевский, В.В. Методические указания к дисциплине «Теория эксплуатационных свойств АТС» [Текст] / В.В. Гаевский, А.М. Иванов. М.: МАДИ, 2007. 53 с.

5. Кнороз, В.И. Шины и колеса [Текст]/ В.И. Кнороз, Е.В. Кленников. М.: Машиностроение, 1975. 184 с.
6. Демьянушко, И.В. Расчет и экспериментальное исследование напряженно-деформированного состояния автомобильных колес на статическую нагрузку [Текст] / И.В. Демьянушко. М.: МАДИ, 2014. 48 с.

7. Ildar Gabitov, Andrei Negovora, Eduard Khasanov, Rustam Galiullin, Mars Farhshatov, Rim Khamaletdinov, Vladimir Martynov, Dmitryi Gusev, Nail Yunusbaev and Mahmut Razyapov, 2019. Risk Reduction of Thermal Damages of Units in Machinery Heat Preparation for Load Acceptance. Journal of Engineering and Applied Sciences, 14: 709-716.

8. Балабин, И.В. Автомобильные и тракторные колеса и шины [Текст] / И.В. Балабин. М.: МГТУ, МАМИ, 2012.920 c. 
9. Зубчанинов, В.Г. Основы тории упругости и пластичности [Текст] / В.Г. Зубчанинов. М.: Высшая школа, 2000. 368 с.

10. Богомолов, В.А. Расчетное распределение давления в пятне контакта шин с поверхностью дорожной одежды [Текст] / В.А. Богомолов // Вестник ХНАДУ. 2016. Вып. 72. С. 143-145.

11. Балакина, Е.Б. Расчет параметров в пятне контакта [Текст] / Е.Б. Балакина // Автомобильная промышленность. 2016. № 3. С. 6-7.

12. Козлов, Ю.Н. Определение взаимного положения зон разного трения в пятне контакта шины с опорной поверхностью [Текст] / Ю.Н. Козлов, В.И. Сальников, А.А. Барашков // Автомобильная промышленность. 2016. № 3. C. 6-7.

13. Зубарев, Н.А. Исследование долговечности дисков автомобильных колес [Текст] / Н.А. Зубарев // Автомобильная промышленность. 1970. № 3. С. 23-25.

14. Демьянушко, И.В. Расчет и экспериментальное исследование напряженно-деформиро- ванного состояния автомобильных колес на статическую нагрузку [Текст] / И.В. Демьянушко, Е.М. Логинов, В.В. Миронова. М.: МАДИ, 2014. $48 \mathrm{c}$.

15. Савельев, Г.В. Колесо для шин с регулируемым давлением [Текст] / Г.В. Савельев // Автомобильный транспорт. 1967. № 5. С. 41-42.

16. Балабин, И.В. Расчет напряженного состояния диска автомобильного колеса [Текст] / И.В. Балабин // Автомобильная промышленность. 2001. № 6. С. 18-20.

17. Балабин, И.В. Расчет напряженного состояния неразъемного обода колеса грузовых автомобилей и автобусов [Текст] / И.В. Балабин, Ю.И. Фомичев, И.С. Чабунин // Автомобильная промышленность. 2003. № 2. С. 29-22.

18. Григорюк, Э.И. Исследование осесимметричного напряженного состояния колес грузовых автомобилей автобусов [Текст] / Э.И. Григорюк, А.Н. Фролов, И.В. Балабин // Автомобильная промышленность. 1964. № 6. C. 27-29.

\section{Сведения об авторах}

1. Максимов Евгений Александрович, кандидат технических наук, доцент, Южно-Уральский государственный аграрный университет, e-mail: Maksimov50@mail.ru.

2. Устиновский Евгений Петрович, кандидат технических наук, профессор, Южно-Уральский государственный аграрный университет.

Проведен анализ различных факторов: давления воздуха в шине, вертикальной нагрузки и типа вылета на изгиб посадочной полки обода трактора и комбайна. Разработана методика расчета влияния вертикальной нагрузки и типа вылета на изгиб посадочной полки обода трактора и комбайна. Показано, что максимальная величина изгиба обода наблюдается для нормаль- ного типа вылета. Для положительного и отрицательного типов вылета величина изгиба обода меньше, чем для нормального. Установлено, что применение положительного и отрицательного вылетов при сборке диска колеса снижает величину максимального изгиба обода по сравнению с изгибом обода при нормальном типе вылета.

E. Maximov, E. Ustinovsky

EFFECT OF THE WHEEL OFFSET

ON THE BEAD SEAT BEND IN FARM MACHINES

Key words: wheel offset type in tractors and combine harvesters; zero offset; negative offset; positive offset; bead seat of the wheel rim.

\section{Authors' personal details}

1. Yevgeny Maksimov, Candidate of Technical Sciences, Associate Professor, South Ural State Agrarian University, e-mail: Maksimov50@mail.ru.

2. Yevgeny Ustinovsky, Candidate of Technical Sciences, Professor, South Ural state agrarian University.

The study analysed the effect of air pressure in the tyre, vertical stress and the wheel offset on the bead seat in tractors and combine-harvesters. As a result, the design procedure was developed for determining the effect of the vertical stress and wheel offset type on the bead seat bend in the tractor and 
combine harvester. The study revealed that the zero offset had the maximum value of the rim bend. The positive and negative offsets had lower values of the rim bend than the zero type. The study found that in

(c) Максимов Е.А., Устиновский Е.П. assembling the wheel disc the positive and negative wheel offsets reduce the value of the maximum rim bend in comparison with the zero type.

УДК 631.171:006.86

DOI: $10.31563 / 1684-7628-2020-54-2-89-94$

Е.В. Малькова, В.Б. Кульневич, Н.И. Олейник

\section{СТАНДАРТИЗАЦИЯ ПРИ ТЕХНИЧЕСКОМ КОНТРОЛЕ ТЕХНОЛОГИЧЕСКИХ ПРОЦЕССОВ В СЕЛЬСКОХОЗЯЙСТВЕННОМ МАШИНОСТРОЕНИИ}

\section{Ключевые слова: стандартизация; технический контроль; разрушающций контроль; техно- логический процесс.}

Введение. Качество выпускаемой продукции обеспечивает конкурентоспособность любого предприятия и зависит от многих факторов, прежде всего от нормативно-технической документации, применяемой на производстве. Технический контроль является одним из основных компонентов управления качеством, основная задача которого - предупреждение выпуска продукции, не соответствующей нормативно-технической документации. Данный процесс осуществим при наличии метрологических служб предприятия и применении современных технических средств контроля. Основными объектами стандартизации систем технического контроля и измерения являются: общие положения, методология, технические средства, организация и управление. Причем в каждом объекте предусматриваются стандарты на терминологию, классификацию, отдельные элементы, отдельные системы и подсистемы.

В современных рыночных условиях производства, занимающиеся массовым выпуском продукции, стремятся к ее диверсификации, периодически переналаживая производство под различную выпускаемую продукцию. Прежде всего это необходимо для устойчивого функционирования производства, так как необходимо изготавливать не только изделия с установленной номенклатурой типоразмеров, но и учитывать поступающие заказы с других предприятий. Соответственно номенклатура выпускаемой продукции значительно увеличивается. Для выполнения данного процесса требуется не только современное и гибко переналаживаемое производственное оборудование, но и соответствующее контрольное техническое оборудование, которое позволит провести проверки и испытания с наибольшей точностью и сделать рациональное заключение о годности изделий.
Анализ важнейших сторон научно-технического прогресса свидетельствует об особом значении стандартизации в решении задачи повышения качества промышленной продукции. Только стандарты могут связать в единое целое, увязать качественные показатели разнообразных изделий, выпускаемых различными отраслями промышленности.

Цели исследования - выработка практических навыков в определении причин возникновения дефектов выпускаемой продукции, овладение методами технического контроля в условиях действующего производства и управления технологическими процессами. На данный момент основная проблема - это недостаток решений по стандартизации на предприятии, то есть с организацией электронных справочников на предприятии или, если более современно, с нормативными ресурсами (НР), куда относятся такие компоненты, как материалы, комплектующие, оборудование, инструмент и т. д.

Материалы и методы исследования. В настоящее время технический контроль рассматривается как равноправный и неотъемлемый элемент системы управления качеством продукции на предприятии.

В своем исследовании мы исходили из того, что для рационального заключения о годности изделий выпускаемой продукции и сокращения числа появления брака на производстве необходимо реализовать виды и методы контроля, направленные на повышение качества выпускаемой продукции.

Так на этапе конструкторско-технологической подготовки на предприятии прежде всего необходимо осуществлять контроль проектирования с применением стандартов, в которых регламентируются терминологическая правильность, определения, относящиеся к организации и управлению СТК, представлены классифика- 